\title{
Transmission Canals for the Photon-Assisted Transport of Electron through the Double-Barrier Resonant Tunneling Structure
}

\author{
M.V. TKaCH*, Ju.O. Seti And O.M. Voitsekhivska \\ Chernivtsi National University, Kotsiybynsky str. 2, Chernivtsi, 58012, Ukraine
}

(Received August 23, 2012; in final form November 20, 2012)

The resonance and non-resonance transmission canals of double-barrier resonant tunneling structure are established for the electron-photon system using the exact solution of one-dimensional non-stationary Schrödinger equation expanded into the Fourier range. It is shown that besides the main and satellite, the mixed quasi-stationary states which cause the appearance of specific transmission canals with the properties strongly dependent on the intensity and frequency of electromagnetic field, exist in the nanostructure.

DOI: 10.12693/APhysPolA.124.94

PACS: 72.10.-d, 73.63.Hs, 68.65.Fg

\section{Introduction}

The electronic transport through the resonant tunneling nanostructures with time-periodical potential, generally, and in the electromagnetic field, particularly, is investigated for a long time both experimentally [1-9] and theoretically [10-18]. The research is caused by the physical phenomena observed in these systems: quantum noise, quantum metastability, negative differential resistance, electron pumps, photon-assisted tunneling, and so on. Also they have an important applied utilization in modern devices, such as super rapid commutators, quantum cascade lasers, and quantum cascade detectors. The theoretical study of photon-assisted transport (PAT) of electrons through the resonant tunneling structures (RTS) driven by strong electromagnetic fields [19-24] is of current interest due to the improvement of physical characteristics of the devices operating in $\mathrm{THz}$ frequency range [6-9].

The theory of nonlinear electronic transport through the systems with time-dependent potentials was developed within different approaches described in detail in reports $[25,26]$. The PAT of electrons through the double-barrier and multi-barrier RTS in high frequency electromagnetic field were studied using the theoretical models which can be divided into two groups.

The first wide group of papers is based on the model tunneling Hamiltonians [14-18, 27, 28], like the one introduced by Bardeen [29], written in the representation of second quantization. In the open system the tunneling Hamiltonian cannot be obtained by the consecutive analytic transition from the coordinate representation to the representation of second quantization because the demanded complete set of wave functions is

*corresponding author; e-mail: ktf@chnu.edu.ua absent. The tunneling Hamiltonians are the model ones and, thus, do not account exactly the boundary conditions at the hetero interfaces and contain time-dependent energies of quasi-particles, practically obtained in the respective closed models. However, they are widely used while studying the nonlinear transport because of the well developed theoretical technique: density and scattering matrix, nonequilibrum Green-Keldysh functions, and other. The main advantage of tunneling Hamiltonian is that it can take into account different dissipative subsystems: phonons, impurities, electron-electron interaction and so on. It gives opportunity to study the ballistic transport of electrons and distinguish the role of dissipative processes in nonlinear transport phenomena, observed at the experiments.

The second group of theoretical papers $[11-15,17,18$, $30-32$ is based at the Hamiltonians obtained in the coordinate representation. They do not contain any fitting parameters and are characterized by the fixed sizes of nanosystems elements, effective masses of electrons and magnitudes of dc- and ac-fields.

The ballistic nonlinear PAT of electrons through the double-barrier RTS and other nanostructures driven by electromagnetic fields is studied within the solution of the complete Schrödinger equation, taking into account the continuity of the wave functions and generalized (when the vector potentials are present) densities of currents at all RTS interfaces. As far as the electromagnetic field is time-dependent, usually, the Floquet method $[25,26]$ is used together with transfer-matrix, $S$-matrix, classic Green's functions, time-dependent perturbation theory, and so on. The one-dimensional model is used as a rule in order to simplify the analytical and numerical calculations considering that the interaction between electrons and electromagnetic field is actual inside of the nanostructure only. The interaction is described within the time-dependent potential: $U(z) \cos \omega t$.

Although the exact solution of the complete Schrödinger equation for the electron in electromag- 
netic field $\boldsymbol{A}(\boldsymbol{r}, t)$ is well known [32] but the problem of nonlinear PAT of electrons through the RTS with the exact accounting of vector potential is complicated enough because it demands to introduce the term: $\frac{1}{2}\left(p_{z}-\frac{e}{c} A_{z}\right) \frac{1}{m(z)}\left(p_{z}-\frac{e}{c} A_{z}\right)$ even in one-dimensional Hamiltonian. However, even the approximated considering of the linear over $A_{z}$ term in the Hamiltonian and one-photon processes gave opportunity to solve the problem of coherent and consecutive tunneling of electrons through the double-barrier RTS correctly [14, 26] and with good correlation to the experiment [2].

The consistent theory of PAT of electrons through the double-, triple-barrier RTS and periodical structures of the Kronig-Penney type in the intensive laser fields with the correct considering of vector potential was developed in the series of papers [19-21]. Solving the complete Schrödinger equation, the authors used the numerical method dividing $z$ region at many intervals and assumed the effective masses $m(z)$, scalar $V(z)$ and vector $A(z, t)$ potentials as constant ones. The matching conditions at the interfaces of all intervals are to be satisfied in order to calculate the probability of tunneling in this approach. It was performed within the transfer-matrix technique. However, the authors mentioned that the numerical calculation of transfer-matrix run against the problem of its instability. It was caused by the very small and very big numbers arising while correctly considering the closed canals.

In this paper we develop the theory of nonlinear PAT of electrons through the double-barrier RTS driven by the electromagnetic field, exactly taking into account the electron-field interaction in the Hamiltonian of the system. We obtain the exact solutions of the complete Schrödinger equation for all parts of the structure, expand the time-dependent wave functions into the Fourier range and take into account the matching conditions at all interfaces of RTS. The proposed approach allows us to study in detail the mixed quasi-stationary states of electron-photon system depending on the energy of electromagnetic field and its driving potential.

\section{Transmission coefficient for the double-barrier RTS driven by electromagnetic field}

We study the transport properties of double-barrier RTS placed into the outer medium. The widths of the both barriers $(\Delta)$ and one of the well $(a)$ are fixed, so the size of RTS is: $L=a+2 \Delta$, Fig. 1 . Index $s=0$, $1,2,3,4$ numbers the different parts of the structure in the Cartesian coordinates with the beginning placed at the left interface of the input barrier and $0 z$ axis perpendicular to the planes of nanofilms. The electron moves along the $0 z$ axis from the left to the right through the double-barrier RTS driven by the homogeneous electromagnetic field. Its characteristics are: the vector potential $A_{z}=\frac{2 c F}{\omega} \sin (\omega t)$, where $c$ is the light velocity, $F$ is the intensity of the electric field, $\omega$ is the frequency of electromagnetic field. In the model of the effective mass and rectangular potential barriers for the electron, we assume that

$$
\begin{aligned}
& m(z)=m_{s}=\left\{\begin{array}{l}
m_{\mathrm{w}}, \\
m_{\mathrm{b}},
\end{array}\right. \\
& U(z)=U_{s}= \begin{cases}0, & s=0,2,4, \\
U, & s=1,3 .\end{cases}
\end{aligned}
$$

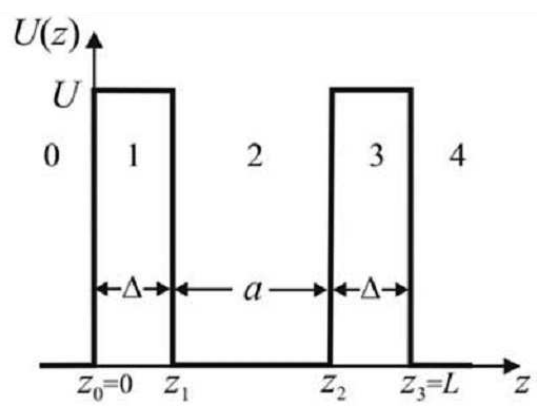

Fig. 1. Potential energy scheme for the electron in double-barrier RTS.

The wave function of the electron is obtained from the time-dependent one-dimensional Schrödinger equation

$$
\begin{aligned}
& \mathrm{i} \hbar \frac{\partial \Psi(z, t)}{\partial t}=\left[-\frac{\hbar^{2}}{2} \frac{\partial}{\partial z} \frac{1}{m(z)} \frac{\partial}{\partial z}+\frac{\mathrm{i} e \hbar A_{z}}{c m(z)} \frac{\partial}{\partial z}\right. \\
&\left.+\frac{e^{2} A_{z}^{2}}{2 c^{2} m(z)}+U(z)\right] \Psi(z, t) .
\end{aligned}
$$

This equation has two linearly independent solutions both for the wells $(s=0,2,4)$ and barriers $(s=1,3)$ :

$$
\begin{aligned}
& \psi_{s}^{ \pm}\left(E, z_{s-1} \leq z \leq z_{s}, \omega, t\right) \\
& \quad=\exp \left( \pm \mathrm{i} k_{s}\left(z-\frac{2 e F}{m_{s} \omega^{2}} \cos (\omega t)\right)\right. \\
& \left.-\frac{\mathrm{i}}{\hbar}\left(E+\frac{(e F)^{2}}{m_{s} \omega^{2}}\left(1-\frac{\sin (2 \omega t)}{2 \omega t}\right)\right) t\right),
\end{aligned}
$$

which describe the forward $\left(\psi_{s}^{+}\right)$and backward $\left(\psi_{s}^{-}\right)$waves with the quantum numbers $k_{s}=$ $\hbar^{-1} \sqrt{2 m_{s}\left(E-U_{s}\right)}$, where $E$ is the electron energy; $z_{-1}=-\infty, z_{4}=\infty$.

The wave function of the electron-photon system $\Psi_{s}(E, z, \omega, t)$ in $s$-th part is the linear superposition of the wave functions $(3)$ :

$$
\begin{aligned}
& \Psi_{s}(E, z, \omega, t)=\sum_{p=-\infty}^{+\infty}\left[B_{s, p}^{+} \psi_{s}^{+}(E+p \Omega, z, \omega, t)\right. \\
& \left.+B_{s, p}^{-} \psi_{s}^{-}(E+p \Omega, z, \omega, t)\right] .
\end{aligned}
$$

Here $\Omega=\hbar \omega$ is the electromagnetic field energy; $p$ is the whole number of photons; $B_{s, p}^{ \pm}$are the time-independent coefficients.

The wave functions $\psi_{s}^{ \pm}(E+p \Omega, z, \omega, t)$ are obtained using the expansion of the time-periodical functions (3) into the exact Fourier ranges

$$
\psi_{s}^{ \pm}(E+p \Omega, z, \omega, t)
$$




$$
\begin{aligned}
& =\mathrm{e}^{ \pm \mathrm{i} k_{s, p} z-\frac{\mathrm{i}}{\hbar}(E+p \Omega) t} \frac{\sin \left(2 \pi \alpha_{s} \beta^{2}\right)}{\pi} \\
& \times \sum_{n, n_{1}, n_{2}=-\infty}^{\infty} \mathrm{i}^{2 n \mp n_{1}} \frac{J_{n_{1}}\left(4 \alpha_{s, p} \beta\right) J_{n_{2}}\left(\alpha_{s} \beta^{2}\right)}{2 \alpha_{s} \beta^{2}+n} \\
& \times \mathrm{e}^{\mathrm{i}\left(n \pm n_{1}+2 n_{2}\right) \omega t},
\end{aligned}
$$

with the convenient dimensionless parameters

$$
\begin{aligned}
& \alpha_{s}=\frac{\hbar^{2}}{2 m_{s} L^{2} \Omega}, \quad \alpha_{s, p}=\alpha_{s} k_{s, p} L, \quad \beta=\frac{e F L}{\Omega}, \\
& k_{s, p}=\hbar^{-1} \sqrt{2 m_{s}\left(E+p \Omega-U_{s}\right)} .
\end{aligned}
$$

After the analytical transformations of formulae (4) and (5), in order to obtain the expansion over harmonics numbered by $N$ only, the $s$-th wave function of the electron-photon system becomes

$$
\begin{aligned}
& \Psi_{s}(E, z, \omega, t)=\mathrm{e}^{-\frac{\mathrm{i}}{\hbar} E t} \sum_{p=-\infty}^{+\infty} \sum_{N=-\infty}^{\infty} \mathrm{e}^{\mathrm{i} N \omega t} \\
& \times\left[B_{s, p}^{+} \mathrm{e}^{\mathrm{i} k_{s, p} z} Q_{N, p}^{+}\left(\alpha_{s, p}, \beta\right)\right. \\
& \left.+B_{s, p}^{-} \mathrm{e}^{-\mathrm{i} k_{s, p} z} Q_{N, p}^{-}\left(\alpha_{s, p}, \beta\right)\right],
\end{aligned}
$$

where

$$
\begin{aligned}
& Q_{N, p}^{ \pm}\left(\alpha_{s, p}, \beta\right)=\frac{\sin \left(2 \pi \alpha_{s} \beta^{2}\right)}{\pi} \\
& \quad \times \sum_{n_{1}, n_{2}=-\infty}^{\infty} \frac{(-1)^{p+N} \mathrm{i}^{ \pm n_{1}} J_{n_{1}}\left(4 \alpha_{s, p} \beta\right) J_{n_{2}}\left(\alpha_{s} \beta^{2}\right)}{2 \alpha_{s} \beta^{2}+p+N \mp n_{1}-2 n_{2}} .
\end{aligned}
$$

All unknown coefficients $B_{s, p}^{ \pm}$are definitely obtained from the conditions of continuity for each harmonic of the complete wave function and their densities of currents taking into account the vector potential of electromagnetic field at four interfaces in any moment of time

$$
\left\{\begin{array}{l}
\Psi_{s}\left(E, z_{s}, \omega, t\right)=\Psi_{s+1}\left(E, z_{s}, \omega, t\right) \quad(s=0 \div 3) \\
\left.\frac{1}{m_{s}}\left(\frac{\partial}{\partial z}-\frac{\mathrm{i} e A_{z}}{c \hbar}\right) \Psi_{s}(E, z, \omega, t)\right|_{z=z_{s}} \\
\quad=\left.\frac{1}{m_{s+1}}\left(\frac{\partial}{\partial z}-\frac{\mathrm{i} e A_{z}}{c \hbar}\right) \Psi_{s+1}(E, z, \omega, t)\right|_{z=z_{s}} .
\end{array}\right.
$$

As far as the electron transports through the main canal $(p=0)$, thus $B_{s=0, p=0}^{+} \neq 0, B_{s=0, p \neq 0}^{+}=0$. Besides, $B_{s=4, p}^{-}=0$ because the reflected waves at the right side out of double-barrier RTS are absent.

We should note that the number of canals $(p)$ and harmonics $(N)$ in the electron-photon system is infinite. Thus, the system of Eqs. (9) contains the infinite number of equations, respectively $B_{s, p}^{ \pm}$coefficients. However, from the expression (8) it is clear that the $Q_{N, p}^{ \pm}$magnitudes decrease when $p$ and $N$ increases, corresponding to the physical considerations. Therefore, in the specific calculations, the system can be confined by the rather big but finite number of equations, determined by the number of harmonics $\left(N_{\max }\right)$ and canals $\left(p_{\max }\right)$ providing the demanded exactness.

Thus, the system of Eqs. (9) is the linearly inhomogeneous one. At the finite number of open canals it allows to obtain all $B_{s, p}^{ \pm}$coefficients expressed throughout
$B_{s=0, p=0}^{+}$. The latter is fixed by the normality condition

$$
\int_{-\infty}^{+\infty} \Psi^{*}\left(k_{0}, z, \omega, t\right) \Psi\left(k_{0}^{\prime}, z, \omega, t\right) \mathrm{d} z=\delta\left(k_{0}-k_{0}^{\prime}\right)
$$

for the wave function of the whole system

$$
\Psi(E, z, \omega, t)=\sum_{s=0}^{4} \Psi_{s}\left(E, z_{s-1} \leq z \leq z_{s}, \omega, t\right) .
$$

Considering the vector potential $\left(A_{z}\right)$, the density of current in any moment of time is written as [19-21]:

$$
\begin{aligned}
& j(E, z, \omega, t)=\frac{\mathrm{i} \hbar}{2 m(z)}\left[\Psi(E, z, \omega, t) \frac{\partial}{\partial z} \Psi^{*}(E, z, \omega, t)\right. \\
& \left.-\Psi^{*}(E, z, \omega, t) \frac{\partial}{\partial z} \Psi(E, z, \omega, t)\right] \\
& -\frac{e}{m(z) c} A_{z}|\Psi(E, z, \omega, t)|^{2}
\end{aligned}
$$

The transmission coefficient for the double-barrier RTS is defined as the ratio between the outcoming density of current $\left(j_{4}^{+}(z=L)\right)$ and that of the falling one $\left(j_{0}^{+}(z=0)\right)$. The electromagnetic field is the periodical one, since it is convenient to study the time-independent transmission coefficient $(D(E, \omega))$, averaging the densities of currents $\left(j_{0}^{+}\right.$and $\left.j_{4}^{+}\right)$over the period of time $(T)$. So, we have

$$
D(E, \omega)=\left\langle j_{4}^{+}(E, \omega)\right\rangle\left\langle j_{0}^{+}(E, \omega)\right\rangle^{-1},
$$
where

$$
\left\langle j_{s}^{+}(E, \omega)\right\rangle=\frac{1}{T} \int_{0}^{T} j_{s}^{+}(E, \omega, t) \mathrm{d} t .
$$

After the analytical calculations we obtain

$$
\begin{aligned}
& D(E, \omega)=D_{0,0}(E, \omega)+\sum_{p \neq 0} D_{p, p}(E, \omega) \\
& \quad+\sum_{p \neq p^{\prime} \neq 0} D_{p, p^{\prime}}(E, \omega) .
\end{aligned}
$$

Here

$$
\begin{aligned}
& D_{0,0}(E, \omega)=\left|B_{0,0}^{+}\right|^{-2}\left|B_{4,0}^{+}\right|^{2}, \\
& D_{p, p^{\prime}}(E, \omega)=(-1)^{p+p^{\prime}}\left|B_{0,0}^{+}\right|^{-2} \\
& \quad \times\left\{k _ { 4 , p } L \operatorname { R e } \left(B_{4, p}^{+}\left(B_{4, p^{\prime}}^{+}\right)^{*} \mathrm{e}^{\mathrm{i} L\left(k_{4, p}-k_{4, p^{\prime}}\right)}\right.\right. \\
& \left.\quad \times \sum_{N=-\infty}^{\infty} Q_{N, p}^{+}\left(\alpha_{0, p}, \beta\right)\left(Q_{N, p^{\prime}}^{+}\left(\alpha_{0, p^{\prime}}, \beta\right)\right)^{*}\right) \\
& -\frac{2 e F L}{\Omega} \operatorname{Im}\left(B_{4, p}^{+}\left(B_{4, p^{\prime}}^{+}\right)^{*} \mathrm{e}^{\mathrm{i} L\left(k_{4, p}-k_{4, p^{\prime}}\right)}\right. \\
& \left.\left.\quad \times \sum_{N=-\infty}^{\infty} Q_{N+1, p}^{+}\left(\alpha_{0, p}, \beta\right)\left(Q_{N, p^{\prime}}^{+}\left(\alpha_{0, p^{\prime}}, \beta\right)\right)^{*}\right)\right\} \\
& \quad \times\left\{\sum _ { N = - \infty } ^ { \infty } \left(k_{0} L\left|Q_{N, 0}^{+}\left(\alpha_{0,0}, \beta\right)\right|^{2}-\frac{2 e F L}{\Omega}\right.\right. \\
& \left.\quad \times \operatorname{Im}\left(Q_{N+1,0}^{+}\left(\alpha_{0,0}, \beta\right)\left(Q_{N, 0}^{+}\left(\alpha_{0,0}, \beta\right)\right)^{*}\right)\right\}^{-1} .
\end{aligned}
$$

The partial contributions into the transmission coefficient 
have the evident physical sense: $D_{0,0}$ is the term arising due to the current over the main canal $(p=0) ; D_{p, p}$ is the term arising due to the current over the $p$-th canal $(p \neq 0) ; D_{p, p^{\prime}}$ is the term arising due to the inter canals $\left(p \neq p^{\prime}\right)$ transitions of electron without any radiation.

The obtained transmission coefficient (15) for the double-barrier RTS allows us to calculate the resonance energies and widths of main and arbitrary number of satellite quasi-stationary states of electron, interacting with electromagnetic field of arbitrary intensity $(F)$ and frequency $(\omega)$. The developed theory proves that this interaction, besides the renormalization of pure quasi-stationary states of electron, brings to the appearance of new mixed states which produce the specific transmission canals of double-barrier RTS.

\section{Properties of the mixed quasi-stationary states of the electron-photon system and transmission canals of double-barrier RTS}

Without the electromagnetic field the spectral parameters: resonance energies $\left(E_{n}\right)$ and resonance widths $\left(\Gamma_{n}\right)$ of electron quasi-stationary states in RTS with the Hamiltonian like: $H=-\frac{\hbar^{2}}{2} \frac{\partial}{\partial z} \frac{1}{m(z)} \frac{\partial}{\partial z}+U(z)$ can be calculated using different techniques [25, 26, 33]: $S$-matrix, probability distribution function, transmission coefficient. The same techniques should be used for the calculation and analysis of quasi-stationary spectrum of electron interacting with electromagnetic field of arbitrary intensity $(F)$ and frequency $(\omega)$. We calculate the resonance energies and width of electron-photon quasi-stationary states in double-barrier RTS using the theory for the transmission coefficient developed in the previous section. We observe the typical, often experimentally [4] and theoretically [26] studied, double-barrier RTS $\left(\operatorname{In}_{0.52} \mathrm{Al}_{0.48} \mathrm{As} / \operatorname{In}_{0.53} \mathrm{Ga}_{0.47} \mathrm{As}\right)$ with known geometrical parameters: $a=9 \mathrm{~nm}, \Delta=3 \mathrm{~nm}$ and physical ones: $U=516 \mathrm{meV}, m_{\mathrm{w}}=0.043 m_{0}, m_{\mathrm{b}}=0.083 m_{0}, m_{0}$ is the mass of pure electron.

In Fig. 2a,b the resonance energies and widths of electron-photon quasi-stationary states are shown as functions of the field energy $(\Omega)$ with such intensity $(F)$ which provides the driving potential energy $\left(U_{L}=\right.$ $e F L=40 \mathrm{meV})$ in the RTS with the size $(L=15 \mathrm{~nm})$. The pattern of the spectrum is complicated but qualitatively just clear because, besides the renormalized main electron quasi-stationary states, the states which are the superpositions of electron ones and the respective number of satellite field harmonics appear due to the interaction with the electromagnetic field.

The analysis of electron-photon spectrum, Fig. 2a, in the plane $(\Omega, E)$ proves that it contains the straight $\left(E=E_{n(p=0)}\right)$ and sloping $\left(E=E_{n(0)}+p \Omega\right)$ plots together with the anticrossings regions. The straight plots $\left(E=E_{n(0)}\right)$ correspond to the resonance energies of $n$-th main quasi-stationary states of electron. They create the main open canals for the electron in double-barrier RTS. The sloping plots $\left(E=E_{n(0)}+p \Omega\right)$ corre-

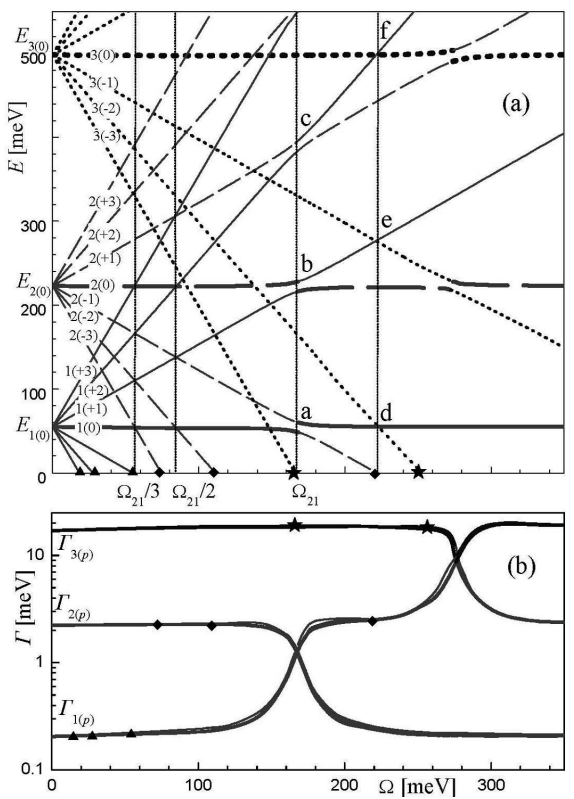

Fig. 2. Resonance energies $E$ (a) and resonance widths $\Gamma$ (b) of main and satellite quasi-stationary states as functions of the electromagnetic field energy $\Omega$. The magnitudes, at which the satellite canals of $n$-th main states become closed and the respective resonance widths do not exist are marked by triangles $(n=1)$, rhombs $(n=2)$ and stars $(n=3)$.

spond to the resonance energies of $p$-th field harmonics of $n$-th electron quasi-stationary state. They create the satellite open canals. The infinite number of positive $(p=+1,+2, \ldots)$ and negative $(p=-1,-2, \ldots)$ satellite quasi-stationary states with the resonance energies $E_{n(p)}=E_{n(0)}+p \Omega$ are observed in the vicinity of field energies $\Omega \leq \Omega_{21}=E_{2}-E_{1}$ near each $n$-th main quasi-stationary state with the energy $E_{n(0)}$.

Denoting the resonance energies of all quasi-stationary states by two indexes $(n(p))$, we adequately characterize the corresponding states of electron-photon system at the straight plots of $E_{n(p)}$ dependence on $\Omega$, even if the resonance energies of some states coincide in the points of anticrossings. However, such indexation becomes insufficient in the vicinity of anticrossings happening when the field energy becomes: $\Omega=\Omega_{n+1, n}=$ $E_{n+1(0)}-E_{n(0)}$. Herein, the superposition of such pairs of quasi-stationary states, with indexes $n(p)$ and $n^{\prime}\left(p^{\prime}\right)$ satisfying the condition $n+p=n^{\prime}+p^{\prime}$ takes place. The peculiar exchange of resonance energies happens between both resonating quasi-stationary states during the anticrossing process. For example, if before the anticrossing $\left(\Omega<\Omega_{n, n^{\prime}}\right)$ the resonance energies satisfy the relationship: $E_{n(p)}>E_{n^{\prime}\left(p^{\prime}\right)}$ then, after it $\left(\Omega>\Omega_{n, n^{\prime}}\right)$ the resonance energies satisfy the reverse one: $E_{n(p)}<E_{n^{\prime}\left(p^{\prime}\right)}$. Thus, in the vicinity of anticrossings the resonance energies of higher quasi-stationary states are conveniently denoted: $E_{n(p), n^{\prime}\left(p^{\prime}\right)}$ and lower ones $-E_{n^{\prime}\left(p^{\prime}\right), n(p)}$ because (as it is clear from Fig. $2 \mathrm{a}$ ) before the anticross- 
ing: $E_{n(p), n^{\prime}\left(p^{\prime}\right)} \rightarrow E_{n(p)}, E_{n^{\prime}\left(p^{\prime}\right), n(p)} \rightarrow E_{n^{\prime}\left(p^{\prime}\right)}$ and after it: $E_{n(p), n^{\prime}\left(p^{\prime}\right)} \rightarrow E_{n^{\prime}\left(p^{\prime}\right)}, E_{n^{\prime}\left(p^{\prime}\right), n(p)} \rightarrow E_{n(p)}$. Consequently, in the proposed denotations for the resonance energies of mixed quasi-stationary states the first pair of indexes characterizes the respective state before the anticrossing and the second one - after it. Further, it would be convenient also for such pairs of mixed states, for which the dependence of transmission coefficient on energy $(E)$ at fixed $\Omega$ is not Lorentz-like but has the shape of curves corresponding to the Fano resonances. Figure 2a proves that the open canals produced by $p$-th satellites of $n$-th main quasi-stationary states become the closed ones in the vicinity of energies satisfying the condition

$$
\Omega \geq \Omega_{n(-|p|)}=E_{n(0)}|p|^{-1} .
$$

In Fig. $2 \mathrm{~b}$ the resonance widths $(\Gamma)$ of quasi-stationary states are presented as functions of the electromagnetic field energy $(\Omega)$. The resonance widths are characterized by the same indexes as the resonance energies: two indexes are used for the region without anticrossing $\left(\Gamma_{n(p)}\right)$, and four - for the region where the anticrossing is present $\left(\Gamma_{n(p), n^{\prime}\left(p^{\prime}\right)}\right)$. In the vicinity of anticrossings, when $\Omega$ tends to the resonance energy $\left(\Omega_{n+1, n}\right)$, the resonance widths of lower $\left(\Gamma_{n(p), n+1(p-1)}\right)$ and higher $\left(\Gamma_{n+1(p-1), n(p)}\right)$ mixed states become closer, i.e. the first one increases and the second - decreases. When $\Omega=\Omega_{n+1, n}$, then $\Gamma_{n(p), n+1(p-1)}=\Gamma_{n+1(p-1), n(p)}=$ $\left(\Gamma_{n(p)}+\Gamma_{n+1(p-1)}\right) / 2$. Herein, the width of anticrossing is fixed by the magnitude: $\Delta E_{n+1(p-1), n(p)}\left(\Omega_{n+1, n}\right)=$ $E_{n+1(p-1)}\left(\Omega_{n+1, n}\right)-E_{n(p)}\left(\Omega_{n+1, n}\right)$. The stronger field causes the decreases of $\Gamma_{n(p), n+1(p-1)}$ tending to $\Gamma_{n(0)}$ and increases of $\Gamma_{n+1(p-1), n(p)}$ tending to $\Gamma_{n+1(0)}$.

In Figs. 2a,b those $\Omega$ values, at which the satellite canals of $n$-th main states become closed and the respective resonance widths do not exist any more are marked by triangles $(n=1)$, rhombs $(n=2)$ and stars $(n=3)$.

In Fig. 3 the transmission coefficient $D$ of double-barrier RTS in the vicinity of main and satellite anticrossings (Fig. 3a,b and $a^{\prime}, b^{\prime}$ ) together with the inter satellite ones (Fig. 3c and $c^{\prime}$ ) is presented as function of energy $(E)$ at the fixed resonance field energy $\left(\Omega_{21}=168 \mathrm{meV}\right)$ and different magnitudes of driving potential $\left(U_{L}\right.$, Fig. $\left.3 \mathrm{a}-\mathrm{c}\right)$ and at fixed driving potential $\left(U_{L}=40 \mathrm{meV}\right)$ and different field energies $(\Omega$, Fig. $\left.3 \mathrm{a}^{\prime}-\mathrm{c}^{\prime}\right)$ in the vicinity of $\Omega_{21}$.

From Fig. 3a it is clear that when the field energy is equal to the resonance one $\left(\Omega=\Omega_{21}\right)$ and anticrossing of energies (point "a" in Fig. 2a) of the first main $|1(0)\rangle$ and first negative satellite of the second main quasi-stationary state $|2(-1)\rangle$ is observed, the transmission coefficient $D(E)$ essentially depends on driving potential $\left(U_{L}\right)$. Without the applied field $\left(U_{L}=0\right), D(E)$ has a shape of the Lorentz function with the resonance energy $E_{1(0)}$ and resonance width $\Gamma_{1(0)}$. When the electromagnetic field is applied and its intensity increases, the shape of the Lorentz curve is deformed, its maximum sharply decreases and, further, $D(E)$ takes shape
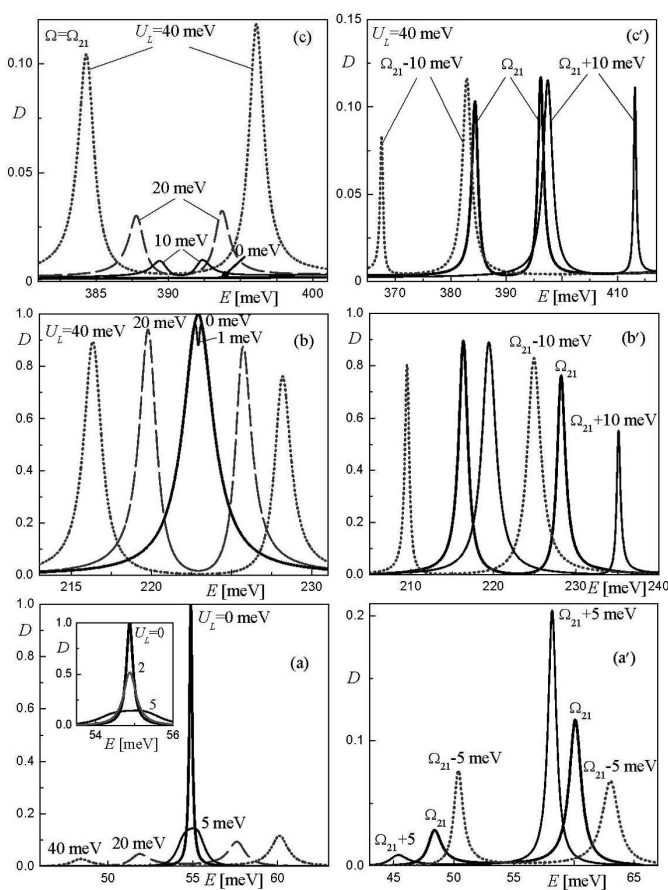

Fig. 3. Transmission coefficient $D$ of double-barrier RTS in the vicinity of main and satellite anticrossings $\left(\mathrm{a}, \mathrm{b}\right.$ and $\left.\mathrm{a}^{\prime}, \mathrm{b}^{\prime}\right)$ together with the inter satellite ones (c and $c^{\prime}$ ) as function of energy $E$ at the fixed resonance field energy $\left(\Omega_{21}=168 \mathrm{meV}\right)$ and different magnitudes of driving potential $U_{L}(\mathrm{a}, \mathrm{b}, \mathrm{c})$ and at fixed driving potential $\left(U_{L}=40 \mathrm{meV}\right)$ and different field energies $\left(\mathrm{a}^{\prime}, \mathrm{b}^{\prime}, \mathrm{c}^{\prime}\right)$ in the vicinity of $\Omega_{21}$.

of two-humped curve with two quasi-Lorentz peaks. Each peak is characterized by its resonance energy and width: $E_{1(0) ; 2(-1)}, \Gamma_{1(0) ; 2(-1)}$ for the lower quasi-stationary state and $E_{2(-1) ; 1(0)}, \Gamma_{2(-1) ; 1(0)}$ for the higher one. The width of anticrossing: $\Delta E_{1(0) ; 2(-1)}=E_{2(-1) ; 1(0)}-E_{1(0) ; 2(-1)}$ increases when the driving potential becomes stronger. The resonance widths of both quasi-stationary states also increase but the maximal magnitude of the transmission coefficient for the higher state increases and for the lower one - decreases. When the both mixed states are split (for example at $U_{L}=40 \mathrm{meV}$, Fig. $3 \mathrm{a}^{\prime}$ ) the increasing field energy $\Omega$ in the vicinity of $\Omega_{21}$ shifts the resonance energies of the pair of quasi-stationary states into the region of smaller energies increasing the transmission coefficient for the higher state and decreasing it for the lower one. The maximal magnitudes of transmission coefficients in both canals produced by the mixed quasi-stationary states are small $(\approx 0.1)$, i.e. they are almost closed.

In Figs. 3b and $b^{\prime}$ one can see the other properties of $D(E)$ function at the anticrossing between the energies of the state $|2(0)\rangle$ and $|1(+1)\rangle$ (point "b" in Fig. 2a). The increasing driving potential, at first, also deforms the curve then, bifurcates it into two quasi-Lorentz peaks making the width of anticrossing bigger. Both these canals are almost open ones. 
Figure $3 \mathrm{c}$ and $\mathrm{c}^{\prime}$ proves that the behavior of $D(E)$ at the anticrossing energies of satellite states $|2(+1)\rangle$ and $|1(+2)\rangle$ (point "c" in Fig. 2a) differs from the previous two cases. Without the field $\left(U_{L}=0\right)$ these states are absent. The transmission coefficient appears as two quasi-Lorentz peaks, Fig. 3c, when $U_{L}$ increases. The width of anticrossing and the height of both peaks also increase. Thus, the transmitting properties of the both satellite canals become better. The both canals shift into the region of bigger energies when the electromagnetic field becomes stronger (Fig. 3c $\mathrm{c}^{\prime}$ ) at fixed driving potential.

Now, we are going to study the properties of transmission coefficient of those groups of mixed quasi-stationary states where the peculiar features of the Fano resonances are observed. The magnitudes of field energies at which these states appear are obtained from the conditions that these field energies are bigger than the minimal resonance one $\left(\Omega_{21}\right)$ and the resonance energy of the first negative $\left(p^{\prime}=-1\right)$ satellite of $n^{\prime}$-th higher state $\left(E_{n^{\prime}\left(p^{\prime}=-1\right)}\right)$ is equal to the energy of the first positive $(p=1)$ satellite of $n$-th lower state $\left(E_{n(p=1)}\right)$. Thus, taking into account that $E_{n(p)}=E_{n(0)}+p \Omega$, we obtain $\Omega_{n^{\prime}(-1) ; n(1)}=\left(E_{n^{\prime}(-1)}-E_{n(1)}\right) / 2$. The minimal magnitude of the field energy $\Omega_{3(-1) ; 1(1)}$, in the vicinity of which such superpositions of quasi-stationary states can appear (at which the transmission coefficient can have the peculiar features of the Fano resonance) corresponds to $\Omega_{31} / 2$. This feature becomes clear from Fig. $2 \mathrm{a}$ and from the abovementioned relationship.

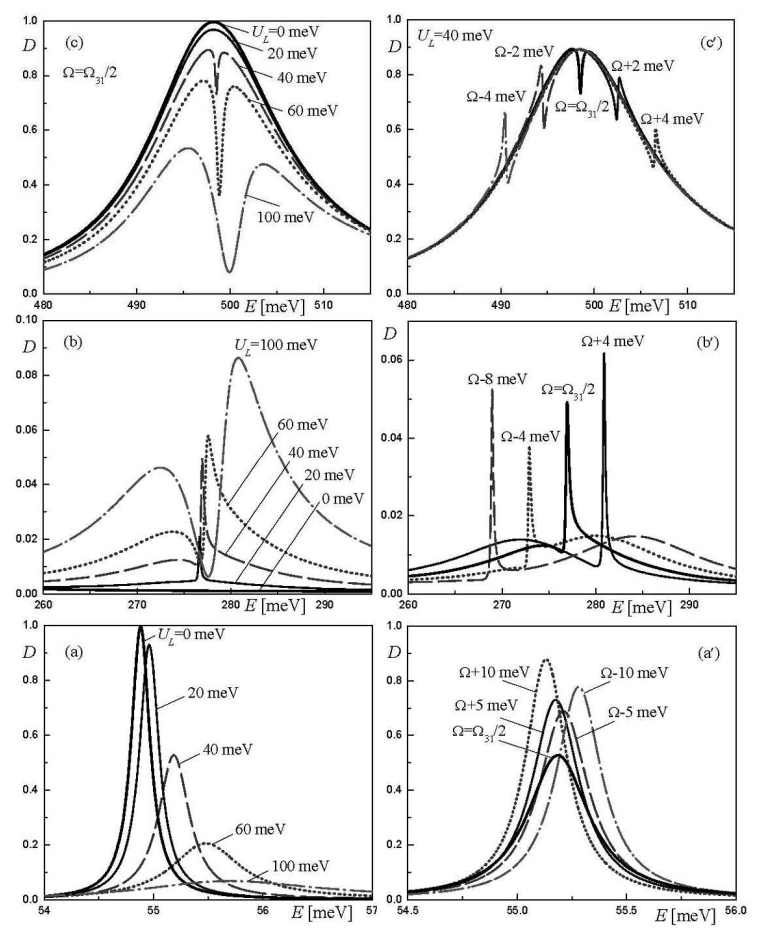

Fig. 4. Transmission coefficient $D$ as function of the electron energy $E$ at the fixed non-resonance energy of the field: $\Omega=\Omega_{31} / 2=221.7 \mathrm{meV}$ and different driving potentials $U_{L}(\mathrm{a}, \mathrm{b}, \mathrm{c})$ and at fixed driving potential $U_{L}=40 \mathrm{meV}$ and different field energies $\Omega\left(\mathrm{a}^{\prime}, \mathrm{b}^{\prime}, \mathrm{c}^{\prime}\right)$.
In Fig. $4 \mathrm{a}-\mathrm{c}$ and $\mathrm{a}^{\prime}-\mathrm{c}^{\prime}$ the transmission coefficient is shown as function of the electron energy $(E)$ at the fixed non-resonance energy of the field: $\Omega=\Omega_{31} / 2=$ $221.7 \mathrm{meV}$ and different driving potentials ( $\mathrm{a}, \mathrm{b}, \mathrm{c}$ ) and at fixed driving potential $U_{L}=40 \mathrm{meV}$ and different field energies $\left(a^{\prime}, b^{\prime}, c^{\prime}\right)$. The calculations were performed for the first three mixed quasi-stationary states (points "d, e, f" in Fig. 2). Figure 4 proves that the shape of the curves $D(E)$ corresponds to the three types of resonances depending on the parameter of asymmetry $(q)$ in the Fano theory [34]: (a) $q \rightarrow \infty$ - quasi-Lorentz curve of the Breit-Wigner type; (b) $0<q<\infty$ - typical Fano resonance; (c) $q=0$ - anti-resonance.

Thus, at the superposition of the first main and second negative harmonics of third quasi-stationary state (Fig. 4a, $\left.\mathrm{a}^{\prime}\right), D(E)$ has a shape of the Breit-Wigner curve with the resonance energies $\left(E_{1(0)}=54.9 \mathrm{meV}\right)$ and resonance widths $\left(\Gamma_{1(0)}=0.2 \mathrm{meV}\right)$ typical for the ground main quasi-stationary state at $U_{L}=0$. At the increasing driving potential, the Lorentz shape of the curve remains but the resonance width increases, transmission maximum decreases and the resonance energy shifts into the region of bigger energies. The stronger field at fixed $U_{L}=40 \mathrm{meV}$ brings to the bigger maximum of transmission coefficient which does not change the resonance energies and widths. Now then, the stronger driving potential at $\Omega_{31} / 2$ decreases the transmission of the canal but the small $\Omega$ varying in the vicinity of $\Omega_{31} / 2$ can improve the transmission properties of double-barrier RTS.

Figure $4 \mathrm{~b}$ and $\mathrm{b}^{\prime}$ shows that the superposition of the states $|1(+1)\rangle$ and $|3(-1)\rangle$ manifests itself in such a way that the shape of transmission coefficient corresponds to the specific asymmetrical Fano resonance with two maxima situated at the opposite sides of the energy: $\left(E_{1(0)}+E_{3(0)}\right) / 2$. At $\Omega=\Omega_{31} / 2$, the increasing driving potential brings to the increase of both maxima and distance between them, too. It is clear that the height of the low-energy peak is smaller than the height of the high-energy one, but both maxima are insignificant (not big), since they produce two canals with small transmission. The both maxima shift into the opposite directions (vicinity of point "e", Fig. 2a) for the bigger field energy $(\Omega)$ at the fixed $U_{L}$.

From Fig. $4 \mathrm{c}, \mathrm{c}^{\prime}$ it is clear that at $\Omega=\Omega_{31} / 2$ the superposition of the states $|1(+2)\rangle$ and $|3(0)\rangle$ manifests itself in such a way, that for the stronger driving potential the Breit-Wigner shape of $D(E)$ gradually deforms into the Fano antiresonance with minimum at $E_{3(0)}$ and width $\Gamma_{1(0)}$ at the top of the Lorentz curve (with resonance energy $E_{3(0)}$ and width $\left.\Gamma_{3(0)}\right)$. Consequently, the transmission coefficient has a shape of two humped almost symmetrical curve with near situated maxima. The distance between them increases at bigger $U_{L}$ and their magnitude decreases. Since, at the field energy $\Omega=\Omega_{31} / 2$ one can see two near situated canals with rather big transmission.

From Fig. $4 c^{\prime}$ it is clear that at the fixed $U_{L} \neq 0$ the varying field energy in the vicinity of $\Omega_{31} / 2$ produces the specific Fano resonance. At bigger $\Omega$, the sharp peak 
with the sharp hollow (having the spectral parameters of $E_{1(2)}, \Gamma_{1(2)}$ order) moves (in accordance to Fig. 2a in the vicinity of point "f") along the wide Breit-Wigner (Lorentz) curve (with spectral parameters $E_{3(0)}, \Gamma_{3(0)}$ ) from low-energy region into the high-energy one, passing the described antiresonance at $\Omega=\Omega_{31} / 2$. Since not only at $\Omega_{31} / 2$ but in its vicinity, too, one can see two near situated canals with big transmission, though the location of their maxima in energy scale does not characterize the resonance energies of quasi-stationary states of the Breit-Wigner type.

The established behavior of mixed quasi-stationary states in double-barrier RTS allow us to analyze the transmission properties of its different canals depending on driving potential $\left(U_{L}\right)$ at different field energies $(\Omega)$. Characterizing the canals by the maximal magnitudes of transmission coefficients $\left(D_{n(p)}=\max D_{n(p)}(E)\right)$ of the respective mixed quasi-stationary states, $D_{n(p)}$ is shown in Fig. 5 as function of $U_{L}$ at three typical magnitudes of field energy: (a) $\Omega=100 \mathrm{meV}$ - the magnitude of non-resonance field energy is smaller than minimal resonance one $\left(\Omega_{21}\right)$; (b) $\Omega=\Omega_{21}=168 \mathrm{meV}-$ minimal resonance energy; (c) $\Omega=\Omega_{31} / 2=221.7 \mathrm{meV}$ - the field energy is equal to the half of the minimal difference between the resonance energies of quasi-stationary states pair with the same parity.

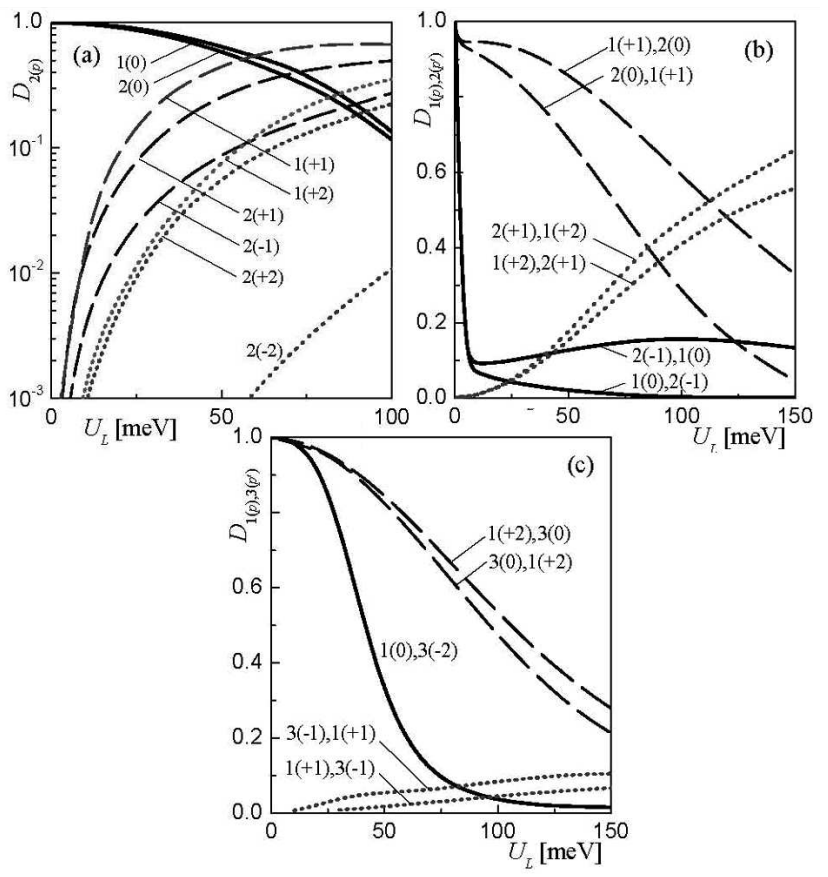

Fig. 5. Maximum of transmission coefficient $(D)$ as function of the driving potential $U_{L}$ at three typical magnitudes of field energies: $\Omega=100 \mathrm{meV}$ - the magnitude of non-resonance field energy is smaller than minimal resonance one (a), $\Omega=\Omega_{21}=168 \mathrm{meV}$ - minimal resonance energy (b), $\Omega=\Omega_{31} / 2=221.7 \mathrm{meV}$ - the field energy is equal to the half of the minimal difference between the resonance energies of quasi-stationary states pair (c).
Figure 5 proves that when the field energy does not coincide with minimal resonance one $\left(\Omega_{21}\right)$ and is smaller, the $U_{L}$ increasing decreases the transmission of both main canals $(|1(0)\rangle,|2(0)\rangle)$ and increases the transmission of their satellite canals $(|1(p)\rangle,|2(p)\rangle)$. Herein, the bigger is the number of the satellite $(p)$ the smaller is the transmission of satellite canal. The canals of positive satellites are considerably more transmitting than the negative ones at the same $|p|$. The transmission of satellite canals becomes bigger than that of the main one at rather strong driving potentials.

When the field energy is equal to the minimal resonance one ( $\Omega=\Omega_{21}$ ), the anticrossings producing the pairs of transmission canals are observed due to the mixing of the pairs of quasi-stationary states at bigger $U_{L}$ (Fig. 5b). The first main quasi-stationary state and first negative satellite of the second main one create the common canal at small $U_{L}$. When $U_{L}$ increases, its transmission at first rapidly decreases till the small magnitude and then the canal bifurcates into pair of canals with small transmission. The transmission of low-energy one insignificantly increases and that of the high-energy one continue to decrease. The second main quasi-stationary state and the first positive satellite of the first main one rapidly produce the pair of canals at bigger $U_{L}$. Their transmission slowly decreases, moreover, for the high-energy - faster and for the low-energy one - slower. The second positive satellite of the main quasi-stationary state and the first positive satellite of the second main one produce the pair of canals. Their transmission increases when $U_{L}$ increases and can approach a considerable magnitude, even bigger than the transmission of the previous pair. Herein, the transmission of the high-energy canal is bigger than that of the low-energy one.

If $\Omega=\Omega_{31} / 2$ (Fig. 5c), the superposition of quasi-stationary states pairs produces one $(|1(0)\rangle,|3(-2)\rangle)$ or two energetically close $(|3(0)\rangle, \quad|1(+2)\rangle$ and $|1(+1)\rangle,|3(-1)\rangle)$ transmission canals at bigger $U_{L}$. The Breit-Wigner-like mixed state $|1(0)\rangle, \quad|3(-2)\rangle$ produces one canal with decreasing transmission. The antiresonance-like mixed state $|3(0)\rangle,|1(+2)\rangle$ produces two energetically close canals with big transmission, slowly decreasing at stronger driving potential. The superposition of two states $|1(+1)\rangle,|3(-1)\rangle$ of satellite type produces the specific Fano resonance creating the two close canals with slowly increasing transmission.

The analyzed properties of PAT of electron through the double-barrier RTS driven by electromagnetic field displayed the new system of transmission canals arising due to the superposition of main electron and satellite field harmonics, on the contrary to the system without the applied electromagnetic field (or very weak one) where the electron quasi-stationary states produced the transmission canals only at the resonance energies.

\section{Conclusions}

We developed the theory for the PAT of electron through the double-barrier RTS within the exact solu- 
tion of non-stationary Schrödinger equation for the electron interacting with electromagnetic field using the expansion of the exact wave function of electron-photon system into the Fourier range over all harmonics.

The mixed quasi-stationary states, being the superpositions of main electron and satellite field harmonics of all orders are observed in the electron-photon system. The properties of all transmission canals of double-barrier RTS essentially depend both on the energy of electromagnetic field and driving potential.

For the stronger driving potential, the transmission of canals at the non-resonance field frequencies (except those, which produce Fano resonances) decreases and that of the satellite ones - increases. At certain magnitude of driving potential, the transmission of satellite canals is bigger than that of the main ones.

The anticrossings between main and satellite states and between the satellite ones happen at the resonance field frequencies, corresponding to the difference between the resonance energies of neighbour main quasi-stationary states when the driving potential increases. Herein, the transmission of the canal produced by the main and satellite states decreases and the transmission of the canals produced by satellite states - increases.

The single or double canals with decreasing transmission are observed at the field energies producing the Fano resonances (e.g., $\Omega_{31} / 2$ ) for the quasi-stationary states being the superpositions of main and satellite ones. The increasing transmission is observed for the canals produced by inter-satellite states.

The established properties of transmission canals of double-barrier RTS, naturally, would be inherent to the multi-barrier RTS, being the basic elements of different nanodevices: quantum cascade lasers, quantum cascade detectors and so on. Thus, the mixed quasi-stationary states are to be taken into account while studying the physical processes in RTS driven by strong electromagnetic fields.

\section{References}

[1] T.C.L.G. Sollner, W.D. Goodhue, P.E. Tannenwald, C.D. Parker, D.D. Peck, Appl. Phys. Lett. 43, 588 (1983).

[2] V.A. Chitta, C. Kutter, R.E.M. de Bekker, J.C. Maan, S.J. Hawksworth, J.M. Chamberlain, M. Henini, G. Hill, J. Phys., Condens. Matter 6, 3945 (1994).

[3] G.B. Serapiglia, E. Paspalakis, C. Sirtori, K.L. Vodopyanov, C.C. Phillips, Phys. Rev. Lett. 84, 1019 (2000).

[4] C.C. Phillips, E. Paspalakis, G.B. Serapiglia, C. Sirtori, K.L. Vodopyanov, Physica E 7, 166 (2000).

[5] E. Kierig, U. Schnorrberger, A. Schietinger, J. Tomkovic, M.K. Oberthaler, Phys. Rev Lett. 100, 190405 (2008).

[6] C. Gmachl, F. Capasso, D.L. Sivco, A.Y. Cho, Rep. Prog. Phys. 64, 1533 (2001).
[7] F.R. Giorgetta, E. Baumann, M. Graf, Q. Yang, C. Manz, K. Kohler, H.E. Beere, D.A. Ritchie, E. Linfield, A.G. Davies, Y. Fedoryshyn, H. Jackel, M. Fischer, J. Faist, D. Hofstetter, IEEE J. Quant. Electron. 45, 1039 (2009).

[8] D. Turčinková, G. Scalari, F. Castellano, M.I. Amanti, M. Beck, J. Faist, Appl. Phys. Lett. 99, 191104 (2011).

[9] S. Sakr, E. Giraud, A. Dussaigne, M. Tchernycheva, N. Grandjean, F.H. Julien, Appl. Phys. Lett. 100, 181103 (2012).

[10] F. Großmann, P. Jung, T. Dittrich, P. Hänggi, Z. Phys. B, Condens. Matter 84, 315 (1991).

[11] M. Wagner, Phys. Rev. B 49, 16544 (1994).

[12] M. Wagner, Phys. Rev. Lett. 76, 4010 (1996).

[13] J. Iñarrea, R. Aguado, G. Platero, Europhys. Lett. 40, 417 (1997).

[14] C. Zhang, Appl. Phys. Lett. 78, 4187 (2001).

[15] S. Kohler, S. Camalet, M. Strass, J. Lehmann, G.-L. Ingold, P. Hänggi, Chem. Phys. 296, 243 (2004).

[16] F.J. Kaisera, P. Hänggi, S. Kohler, Eur. Phys. J. B 54, 201 (2006).

[17] K.-H. Yang, C.-Z. Liu, Eur. Phys. J. B 72, 409 (2009).

[18] J. Hammer, W. Belzig, Phys. Rev. B 84, 085419 (2011).

[19] E. Saczuk, J.Z. Kamiński, Phys. Status. Solidi B 240, 603 (2003).

[20] F.H.M. Faisal, J.Z. Kamiński, E. Saczuk, Phys. Rev. A 72, 023412 (2005).

[21] F.H.M. Faisal, J.Z. Kamiński, E. Saczuk, Laser Phys. 16, 272 (2006).

[22] Li Chun-Lei, Xu Yan, Chin. Phys. B 19, 057202 (2010).

[23] A.B. Pashkovskii, JETP Lett. 93, 559 (2011).

[24] N.V. Tkach, Yu.A. Seti, JETP Lett. 95, 271 (2012).

[25] M. Grifoni, P. Hänggi, Phys. Rep. 304, 229 (1998).

[26] G. Platero, R. Aguado, Phys. Rep. 395, 1 (2004).

[27] A.-P. Jauho, N.S. Wingreen, Y. Meir, Phys. Rev. B 50, 5528 (1994).

[28] Ph. Brune, C. Bruder, H. Schoeller, Phys. Rev. B 56, 4730 (1997).

[29] J. Bardeen, Phys. Rev. Lett. 6, 57 (1961).

[30] J. Iñarrea, G. Platero, C. Tejedor, Phys. Rev. B 50 , 4581 (1994).

[31] G. Murillo, P.A. Schulz, J.C. Arce, Appl. Phys. Lett. 98, 102108 (2011).

[32] C. Zhang, Phys. Rev. B 66, 081105(R) (2002).

[33] N.V. Tkach, Yu.A. Seti, Low Temp. Phys. 35, 556 (2009).

[34] U. Fano, Phys. Rev. 124, 1866 (1961). 